\title{
The Gumbel-Lomax Distribution: Properties and Applications
}

\author{
M. H. Tahir ${ }^{1, *}$, M. Adnan Hussain ${ }^{1}$, Gauss M. Cordeiro ${ }^{2}$, G.G. Hamedani ${ }^{3}$, M. Mansoor ${ }^{1}$ and M. Zubair ${ }^{1,4}$ \\ ${ }^{1}$ Department of Statistics, The Islamia University of Bahawalpur, Bahawalpur-63100, Pakistan; \\ ${ }^{2}$ Department of Statistics, Federal University of Pernambuco, Recife, PE, Brazil; \\ ${ }^{3}$ Department of Mathematics, Statistics and Computer Science, Marquette University, Milwaukee, USA \\ ${ }^{4}$ Department of Statistics, Government Degree College Khairpur Tamewali, Pakistan; \\ E-mails:mtahirstat@gmail.com; adnanidc@gmail.com; gauss@de.ufpe.br; gholamhoss.hamedani@marquette.edu; \\ mansoor.abbasi143@gmail.com; zubair.stat@yahoo.com
}

Received 2 October 2014

Accepted 15 July 2015

\begin{abstract}
We introduce a new four-parameter model called the Gumbel-Lomax distribution arising from the Gumbel$X$ generator recently proposed by Al-Aqtash (2013). Its density function can be right-skewed and reversed-J shaped, and can have decreasing and upside-down bathtub shaped hazard rate. Various structural properties of the new distribution are obtained including explicit expressions for the quantile function, ordinary and incomplete moments, Lorenz and Bonferroni curves, mean residual lifetime, mean waiting time, probability weighted moments, generating function and Shannon entropy. We also provide the density function for the order statistics. Some characterizations of the new distribution based on the conditional expectations of certain functions of the random variable are also proposed. The model parameters are estimated by the method of maximum likelihood and the observed information matrix is determined. The flexibility of the new model is illustrated by means of two real lifetime data sets.
\end{abstract}

Keywords: Characterization; Gumbel distribution; Gumbel-X family; Lomax distribution; Shannon entropy; T-X family.

2000 Mathematics Subject Classification: 60E05, 62E10, 62N05

\section{Introduction}

Many lifetime distributions have been constructed with a view for applications in several areas, in particular, survival analysis, reliability engineering, demography, actuarial study, hydrology and others. Generalizing continuous univariate distributions is an old practice by introducing additional parameters such as location, scale, shape and inequality in the distribution and then seeing changes in its shape. The recent revolution, however, has proved that the introduction of shape parameters is essential to better explore the tails and other properties of the distribution. Following the latest trend, applied statisticians are now able to obtain more improved results since the generalized distribution provides better goodness-of-fit to the data sets rather than following old-fashion strategy. Although, Azzalini (1985), Marshall and Olkin (1997) and Gupta et al. (1998) were the pioneers in this area, the revolutionary work starts after introducing the beta generator (beta-G) defined by Eugene et

$\overline{{ }^{*} \text { Corresponding author. }}$ 
al. (2002) and subsequently by Jones (2004). Later, other competing generators were introduced as the Kumaraswamy-G by Cordeiro and de Castro (2011) and McDonald-G by Alexander et al. (2012). Practitioner and applied researchers enjoyed exploring some probability models by comparing their performances with other G-classes of distributions. Similar works were proposed based on the gamma, Weibull, Kumaraswamy type 2, logistic, Lomax and generalized-exponential (GE) distributions: Viz. Zografos and Balakrishnan (2009) [Gamma-G(type 1)] and [Weibull-G (type 1)], Ristić and Balakrishnan (2012) [Gamma-G (type 2)], Torabi and Montazri (2012) [Odd Gamma-G (type 3)], Cordeiro et al. (2013) [exponentiated-generalized-G], Bourguignion et al. (2014) [Odd Weibull-G (type 2)], Amini et al. (2014) [Gamma-G (type 1 and 2)], Torabi and Montazri (2014) [Logistic-G], Cordeiro et al. (2014) [Lomax-G], Tahir et al. (2015a) [Logistic-X], Tahir et al. (2015b) [Odd generalized-exponential-G] and Tahir et al. (2015c) [Weibull-G (type 3)].

An advancement in G-classes revolution begin with the seminal work of Alzaatreh (2011) and Alzaatreh et al. (2013) when they defined the transformed (T)-transformer $(X)$ family (for short, $T$ - $X$ family). Alzaatreh et al. (2013) studied two main classes of generated models: gamma-X and Weibull-X.

Let $r(t)$ be the probability density function (pdf) of a random variable $T \in[a, b]$ for $-\infty<$ $a<b<\infty$ and let $F(x)$ be the cumulative distribution function (cdf) of a random variable $X$ such that the link function $W(\cdot):[0,1] \longrightarrow[a, b]$ satisfies two conditions: (i) $W(\cdot)$ is differentiable and monotonically non-decreasing, and (ii) $W(x) \rightarrow a$ as $x \rightarrow-\infty$ and $W(x) \rightarrow b$ as $x \rightarrow \infty$.

Alzaatreh et al. (2013) defined the cdf of the $T$ - $X$ family by

$$
F(x)=\int_{a}^{W[G(x)]} r(t) d t,
$$

where $W[G(x)]$ satisfies the above conditions.

The pdf corresponding to (1.1) is given by

$$
f(x)=\frac{d W[G(x)]}{d x} r[W(G(x))] .
$$

Following Alzaatreh et al. (2013), Tahir et al. (2015a) defined and studied the Logistic- $X$ family of distributions.

Recently, Al-Aqtash (2013) and Al-Aqtash et al. $(2014,2015)$ proposed the Gumbel-X family by taking $T$ as the Gumbel random variable in (1.1), namely

$$
F(x)=\exp \left\{-\mathrm{e}^{\mu / \sigma}\left[\frac{\mathrm{G}(\mathrm{x})}{1-\mathrm{G}(\mathrm{x})}\right]^{-1 / \sigma}\right\}
$$

where $0<x<\infty, \sigma, \alpha, \lambda>0$ and $-\infty<\mu<\infty$.

Setting $\lambda=e^{\mu / \sigma}$ throughout the manuscript to avoid non-identifiability problem, the cdf (1.3) reduces to

$$
F(x)=\exp \left\{-\lambda\left[\frac{G(x)}{1-G(x)}\right]^{-1 / \sigma}\right\} .
$$

The pdf corresponding to (1.4) is given by

$$
f(x)=\frac{\lambda h(x)}{\sigma G(x)}\left[\frac{G(x)}{1-G(x)}\right]^{-1 / \sigma} \exp \left\{-\lambda\left[\frac{G(x)}{1-G(x)}\right]^{-1 / \sigma}\right\},
$$

where $X$ is any continuous random variable with cdf $F(x)$ and hazard rate function (hrf) $h(x)$. 
The rest of the paper unfolds as follows. In Section 2, we define the Gumbel-Lomax ("GuLx" for short) distribution. In Section 3, we provide the shapes of the density and hazard rate function (hrf). A useful expansion for the GuLx density is presented in Section 4. In Section 5, some statistical properties of the new distribution are explored such as the quantile function (qf), ordinary and incomplete moments, Bonferroni and Lorenz curves, mean deviations, mean residual life, mean waiting time, moment generating function (mgf), probability weighted moments (PWMs), Shannon entropy and order statistics. In Section 6, we provide some characterizations of the GuLx distribution. In Section 7, the model parameters are estimated using the maximum likelihood method and the information matrix is determined. In Section 8, we explore the usefulness of the new model by means of two applications. Finally, Section 9 offers some concluding remarks.

\section{The Gumbel-Lomax distribution}

A random variable $Z$ has the Lomax distribution with two parameters $\alpha$ and $\beta$, if its cdf is given by

$$
G_{\alpha, \beta}(x)=1-\left(1+\frac{x}{\beta}\right)^{-\alpha}, \quad x>0, \quad \alpha, \beta>0 .
$$

The pdf and hrf corresponding to (2.1) are

$$
g_{\alpha, \beta}(x)=\frac{\alpha}{\beta}\left(1+\frac{x}{\beta}\right)^{-(\alpha+1)}
$$

and

$$
h_{\alpha, \beta}(x)=\frac{\alpha}{\beta}\left(1+\frac{x}{\beta}\right)^{-1} \text {. }
$$

From now on, we can omit the dependence on the model parameters. Inserting (2.1) and (2.3) in (1.4) and (1.5), the cdf and pdf of the four-parameter GuLx distribution are given by

$$
F(x)=\exp \left\{-\lambda\left[\left(1+\frac{x}{\beta}\right)^{\alpha}-1\right]^{-1 / \sigma}\right\}
$$

and

$$
f(x)=\frac{\alpha \lambda\left(1+\frac{x}{\beta}\right)^{-\frac{\alpha}{\sigma}-1}}{\beta \sigma\left\{1-\left(1+\frac{x}{\beta}\right)^{-\alpha}\right\}^{\frac{1}{\sigma}+1}} \exp \left\{-\lambda\left[\left(1+\frac{x}{\beta}\right)^{\alpha}-1\right]^{-1 / \sigma}\right\},
$$

respectively. Henceforth, a random variable $X$ with density function (2.5) is denoted by $X \sim \operatorname{GuLx}(\alpha, \beta, \lambda, \sigma)$.

The survival function (sf), hrf and cumulative hazard rate function (chrf) are, respectively, given by

$$
\begin{gathered}
S(x)=1-\exp \left\{-\lambda\left[\left(1+\frac{x}{\beta}\right)^{\alpha}-1\right]^{-1 / \sigma}\right\}, \\
h(x)=\frac{\alpha \lambda\left(1+\frac{x}{\beta}\right)^{-\frac{\alpha}{\sigma}-1} \exp \left\{-\lambda\left[\left(1+\frac{x}{\beta}\right)^{\alpha}-1\right]^{-1 / \sigma}\right\}}{\beta \sigma\left\{1-\left(1+\frac{x}{\beta}\right)^{-\alpha}\right\}^{\frac{1}{\sigma}+1}\left[1-\exp \left\{-\lambda\left[\left(1+\frac{x}{\beta}\right)^{\alpha}-1\right]^{-1 / \sigma}\right\}\right]}, \\
\text { Published by Atlantis Press } \\
\text { Copyright: the authors }
\end{gathered}
$$


and

$$
H(x)=-\log \left[1-\exp \left\{-\lambda\left[\left(1+\frac{x}{\beta}\right)^{\alpha}-1\right]^{-1 / \sigma}\right\}\right] .
$$

\section{Shapes of the density and hazard rate function}

The shapes of the density and hrf can be described numerically. The critical points of the GuLx density are the roots of the equation:

$$
\sigma\left\{\left(\frac{\beta+x}{\beta}\right)^{\alpha}-1\right\}+\alpha\left\{\sigma-\lambda\left(\frac{\beta+x}{\beta}\right)^{\alpha}\left[\left\{\left(\frac{\beta+x}{\beta}\right)^{\alpha}-1\right\}^{-1 / \sigma}\right]+\left(\frac{\beta+x}{\beta}\right)^{\alpha}\right\}=0 .
$$

The critical points of the GuLx hrf are obtained from the equation

$$
\begin{aligned}
& \left(\frac{\beta+x}{\beta}\right)^{\alpha}\left[e^{\lambda\left\{\left(\frac{\beta+x}{\beta}\right)^{\alpha}-1\right\}^{-1 / \sigma}}\left\{\alpha \lambda-(\alpha+\sigma)\left\{\left(\frac{\beta+x}{\beta}\right)^{\alpha}-1\right\}^{1 / \sigma}\right\}\right. \\
& \left.+(\alpha+\sigma)\left\{\left(\frac{\beta+x}{\beta}\right)^{\alpha}-1\right\}^{1 / \sigma}\right] \\
& -\sigma(\alpha-1)\left\{\left(\frac{\beta+x}{\beta}\right)^{\alpha}-1\right\}^{1 / \sigma}\left[e^{\left.\lambda\left\{\left(\frac{\beta+x}{\beta}\right)^{\alpha}-1\right\}^{-1 / \sigma}-1\right]=0 .}\right.
\end{aligned}
$$

By using any numerical software, we can examine equations (3.1) and (3.2) to determine the local maximum and minimum and inflexion points.

Figures 1 and 2 display some plots of the pdf and hrf of the GuLx distribution for some parameter values. Figure 1 indicates that the GuLx density is right-skewed and reversed-J shaped. Figure 2 reveals that the GuLx hrf can have decreasing and upside-down bathtub shapes.

(a)

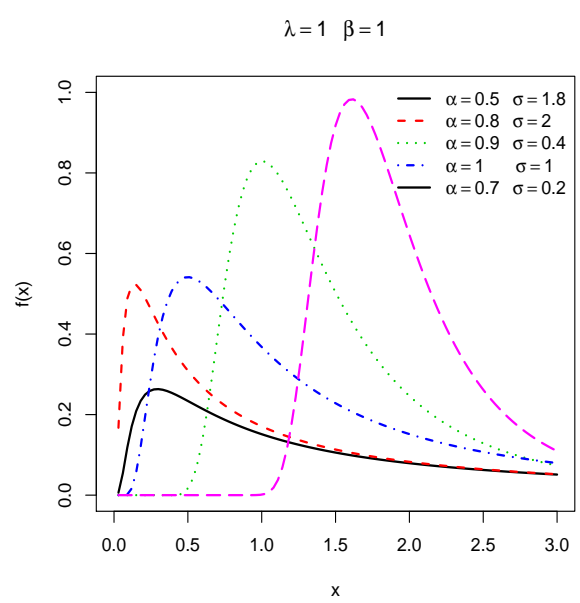

(b)

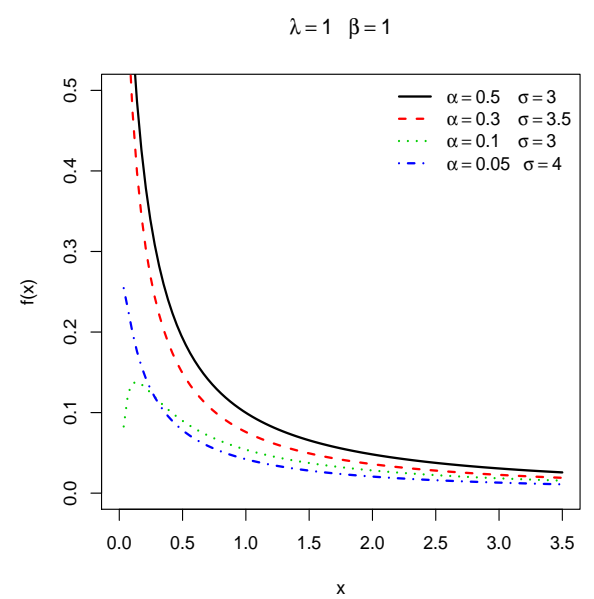

Fig. 1. Plots of the GuLx density for some parameter values. 
(a)

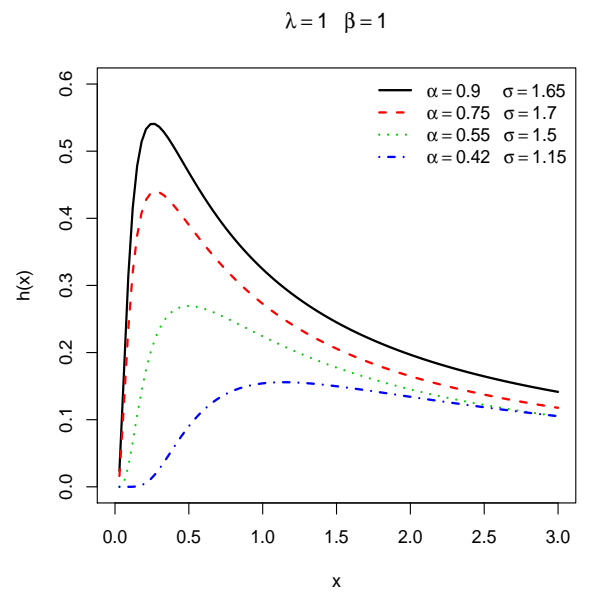

(b)

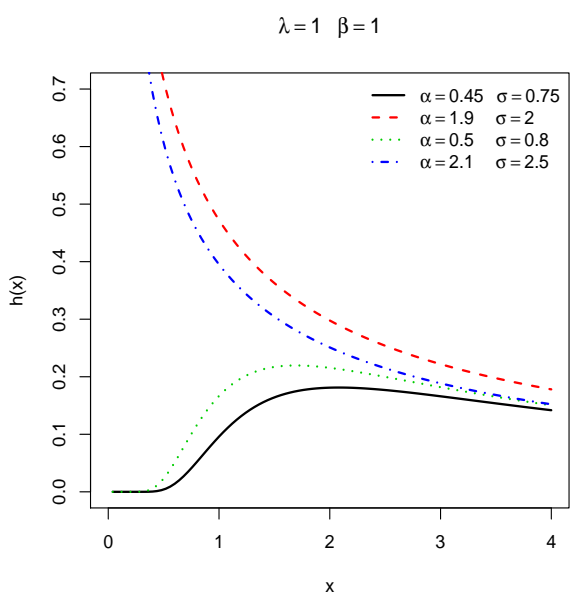

Fig. 2. Plots of GuLx hazard rate for some parameter values.

\section{Mixture representation}

Inserting (2.1) in equation (1.5), we have

$$
f(x)=\frac{\alpha \lambda\left(1+\frac{x}{\beta}\right)^{-\frac{\alpha}{\sigma}-1}}{\beta \sigma\left\{1-\left(1+\frac{x}{\beta}\right)^{-\alpha}\right\}^{\frac{1}{\sigma}+1}} \underbrace{\exp \left\{-\lambda\left[\left(1+\frac{x}{\beta}\right)^{\alpha}-1\right]^{-1 / \sigma}\right\}}_{A} .
$$

By expanding the quantity $A$ in power series,

$$
A=\sum_{i=0}^{\infty} \frac{(-1)^{i} \lambda^{i}}{i !}\left\{\left(1+\frac{x}{\beta}\right)^{\alpha}-1\right\}^{-i / \sigma} .
$$

Combining the last two results, we can write

$$
f(x)=\frac{\alpha \lambda^{(i+1)} \sum_{i=0}^{\infty}\left(1+\frac{x}{\beta}\right)^{-\frac{\alpha(i+1)}{\sigma}-1}}{\beta \sigma\left\{1-\left(1+\frac{x}{\beta}\right)^{-\alpha}\right\}^{\frac{(i+1)}{\sigma}+1}} .
$$

By expanding the quantity $B=\left\{1-\left(1+\frac{x}{\beta}\right)^{-\alpha}\right\}^{\frac{(i+1)}{\sigma}+1}$ in power series such that $\left(1+\frac{x}{\beta}\right)<1$, we obtain

$$
B=\sum_{j=0}^{\infty}(-1)^{j}\left(\begin{array}{c}
-\frac{(i+1)}{\sigma}-1 \\
j
\end{array}\right)\left(1+\frac{x}{\beta}\right)^{-\alpha j} .
$$

Combining the last two results, we have

$$
f(x)=\sum_{i, j=0}^{\infty} \underbrace{\frac{(-1)^{i+j} \lambda^{(i+1)}}{i ![(i+1)-\sigma j]}\left(\begin{array}{c}
-\frac{(i+1)}{\sigma}-1 \\
j
\end{array}\right)}_{V_{i, j}} \underbrace{\frac{\alpha[(i+1)+\sigma j]}{\beta \sigma}\left(1+\frac{x}{\beta}\right)^{-\frac{\alpha}{\sigma}((i+1)+\sigma j)-1}}_{g_{\alpha[(i+1)+\sigma j] / \sigma, \beta}(x)} .
$$


M.H. Tahir et al.

In a more simplified form, the pdf of $X$ can be expressed as

$$
f(x)=\sum_{i, j=0}^{\infty} V_{i, j} g_{\alpha[(i+1)+\sigma j] / \sigma, \beta}(x) .
$$

Equation (4.1) reveals that the GuLx density function has a mixture representation of Lomax densities with parameters $\alpha[(i+1)+\sigma j] / \sigma$ and $\beta$. Thus, several of its structural properties can be derived from those of the Lomax distribution. The coefficients $V_{i, j}$ depend only on the generator parameters. Equation (4.1) is the main result of this section.

\section{Some structural properties}

\subsection{Transformation}

The relationship between the GuLx and Gumbel distributions is given in the following lemma.

Lemma 5.1. If $Y$ is a random variable having the Gumbel distribution with parameters $\mu$ and $\sigma$, then the random variable $X=\beta\left\{\left(1+\mathrm{e}^{Y}\right)^{1 / \alpha}-1\right\}$ has the GuLx distribution.

\subsection{Quantile function and simulation}

The qf of a distribution has many uses in both the theory and applications in Statistics. The qf of $X$ is obtained by inverting (2.4) as

$$
x=Q(u)=\beta\left\{\left[1+\left(-\frac{1}{\lambda} \log (u)\right)^{-\sigma}\right]^{1 / \alpha}-1\right\}, \quad 0<u<1 .
$$

If $U$ has a uniform distribution in $(0,1)$, then $X=Q(U)$ has the density (2.5).

\subsection{Moments}

Theorem 5.1. The rth ordinary moment of the GuLx distribution is

$$
\mu_{r}^{\prime}=\mathbb{E}\left(X^{r}\right)=\beta^{r} \sum_{j, k=0}^{\infty} W_{j, k, r} \Gamma(1-\sigma k), \quad \sigma k>0
$$

where $W_{j, k, r}=(-1)^{j} \lambda^{\sigma k}\left(\begin{array}{c}r \\ j\end{array}\right)\left(\begin{array}{c}(r-j) / \alpha \\ k\end{array}\right)$ and $\Gamma(a)=\int_{0}^{\infty} t^{a-1} \mathrm{e}^{-\mathrm{t}} \mathrm{dt}$ is the gamma function.

Proof. By using Lemma (5.1),

$$
\mathbb{E}\left(X^{r}\right)=\frac{\beta^{r} \lambda}{\sigma} \int_{-\infty}^{\infty} \underbrace{\left\{\left(1+\mathrm{e}^{y}\right)^{1 / \alpha}-1\right\}^{r}}_{C} \mathrm{e}^{-\frac{\mathrm{y}}{\sigma}} \mathrm{e}^{-\lambda \mathrm{e}^{-\frac{\mathrm{y}}{\sigma}}} \mathrm{dy} .
$$

Consider the quantity $C$ in the last equation

$$
C=\left\{\left(1+\mathrm{e}^{y}\right)^{1 / \alpha}-1\right\}^{r}=\frac{\left\{1-\left(1+\mathrm{e}^{y}\right)^{-1 / \alpha}\right\}^{r}}{\left(1+\mathrm{e}^{y}\right)^{-r / \alpha}} .
$$


Using the power series for $(1-z)^{a}$ and the binomial expansion in $C$, we obtain

$$
C=\sum_{j, k=0}^{\infty}(-1)^{j}\left(\begin{array}{l}
r \\
j
\end{array}\right)\left(\begin{array}{c}
(r-j) / \alpha \\
k
\end{array}\right) \mathrm{e}^{y k} .
$$

The result (5.2) follows after substituting (5.4) in (5.3) and noting that

$$
\int_{-\infty}^{\infty} \mathrm{e}^{\mathrm{yk}} \mathrm{e}^{-\frac{\mathrm{y}}{\sigma}} \mathrm{e}^{-\lambda \mathrm{e}^{-\frac{\mathrm{y}}{\sigma}}} \mathrm{dy}=\lambda^{\sigma \mathrm{k}-1} \Gamma(1-\sigma \mathrm{k}) .
$$

Setting $r=1$ in (5.2), the mean of $X$ reduces to

$$
\mu_{1}^{\prime}=\beta \sum_{j, k=0}^{\infty} W_{j, k, 1} \Gamma(1-\sigma k) .
$$

Further, the central moments $\left(\mu_{r}\right)$ and cumulants $\left(\kappa_{r}\right)$ of $X$ are obtained from (5.2) as

$$
\mu_{r}=\sum_{k=0}^{n}(-1)^{k}\left(\begin{array}{l}
r \\
k
\end{array}\right) \mu_{1}^{\prime k} \mu_{r-k}^{\prime} \quad \text { and } \quad \kappa_{r}=\mu_{n}^{\prime} \sum_{k=1}^{r-1}\left(\begin{array}{c}
r-1 \\
k-1
\end{array}\right) \kappa_{k} \mu_{r-k}^{\prime} .
$$

respectively, where $\kappa_{1}=\mu_{1}^{\prime}$. The skewness and kurtosis measures can be calculated from the ordinary moments using well-known relationships. The $n$th descending factorial moment of $X$ (for $n=1,2, \ldots)$ is

$$
\mu_{r}^{\prime}=E\left[X^{(r)}\right]=E[X(X-1) \times \cdots \times(X-r+1)]=\sum_{j=0}^{r} s(r, j) \mu_{j}^{\prime},
$$

where $s(r, j)=(j !)^{-1}\left|d^{j} j^{(r)} / d x^{j}\right|_{x=0}$ is the Stirling number of the first kind.

\subsection{Incomplete moments}

Theorem 5.2. The rth incomplete moment of $X$, say $m_{r}(y)=\int_{0}^{y} x^{r} f(x) d x$, is given by

$$
\begin{gathered}
m_{r}(y)=\beta^{r} \lambda^{\sigma k} \sum_{j, k=0}^{\infty} W_{j, k}\left[\frac{\lambda^{-1-\sigma k} z^{-1-\sigma k}(\lambda z)^{1 / 2 \sigma k} \mathrm{e}^{-1 / 2 \lambda z} \mathbf{M}(-1 / 2 \sigma k,-1 / 2 \sigma k+1 / 2, \lambda z)}{\sigma k-1}\right. \\
\left.+\frac{\pi \csc (\pi \sigma k)}{\Gamma(\sigma k) \lambda Z}\right]
\end{gathered}
$$

Proof. By using Maple (for $z>0$ ), we obtain

$$
\begin{gathered}
\int_{z}^{\infty} z^{-\sigma k} \mathrm{e}^{-\lambda \mathrm{z}} \mathrm{dz}=z \lambda^{\sigma k}\left[\frac{\lambda^{-1-\sigma k} z^{-1-\sigma k}(\lambda z)^{1 / 2 \sigma k} \mathrm{e}^{-1 / 2 \lambda z} \mathbf{M}(-1 / 2 \sigma k,-1 / 2 \sigma k+1 / 2, \lambda z)}{\sigma k-1}\right. \\
\left.+\frac{\pi \csc (\pi \sigma k)}{\Gamma(\sigma k) \lambda z}\right],
\end{gathered}
$$

where $\mathbf{M}(a, b, c)=\frac{c^{b+1 / 2}{ }_{1} F_{1}(1 / 2-a+b ; 1+2 b ; c)}{\mathrm{e}^{1 / 2 c}}$ is the WhittakerM function. 
The main application of the first incomplete moment refers to the Bonferroni and Lorenz curves. These curves are very useful in several fields. For a given probability $\pi$, they are defined by $B(\pi)=$ $m_{1}(q) /\left(\pi \mu_{1}^{\prime}\right)$ and $L(\pi)=m_{1}(q) / \mu_{1}^{\prime}$, respectively, where $q=Q(\pi)$ is determined from (5.1) and $m_{1}(q)$ comes from (5.5) with $r=1$.

The amount of scatter in a population is measured to some extent by the totality of deviations from the mean and median defined by $\delta_{1}=\int_{0}^{\infty}|x-\mu| f(x) d x$ and $\delta_{2}(x)=\int_{0}^{\infty}|x-M| f(x) d x$, respectively, where $\mu_{1}^{\prime}=E(X)$ is the mean and $M=Q(0.5)$ is the median. These measures can be expressed as $\delta_{1}=2 \mu_{1}^{\prime} F\left(\mu_{1}^{\prime}\right)-2 m_{1}\left(\mu_{1}^{\prime}\right)$ and $\delta_{2}=\mu_{1}^{\prime}-2 m_{1}(M)$, where $F\left(\mu_{1}^{\prime}\right)$ comes from (2.4) and $m_{1}(\cdot)$ is given by (5.5) with $r=1$.

Further applications of the first incomplete moment are related to the mean residual life and mean waiting time given by $m(t)=\left[1-m_{1}(t)\right] / S(t)-t$ and $\mu(t)=t-\left[m_{1}(t) / F(t)\right]$, respectively, where $F(t)$ and $S(t)=1-F(t)$ are obtained from (2.4).

\subsection{Probability weighted moments}

The $(r, q)$ th PWM of $X$ (for $r \geq 1, q \geq 0$ ) is formally defined by

$$
\rho_{r, q}=E\left[X^{r} F(X)^{q}\right]=\int_{0}^{\infty} x^{r} F(x)^{q} f(x) d x .
$$

For some specific distributions, the relations between the PWMs and the parameters have simple analytical structure than those between the conventional moments and the parameters. Let

$$
F(x)^{q}=\left[\exp \left\{\left[-\lambda\left\{\left(1+\frac{x}{\beta}\right)^{\alpha}-1\right\}^{-1 / \sigma}\right]\right\}\right]^{q} \text {. }
$$

Expanding $F(x)^{q}$ in power series, we have

$$
F(x)^{q}=\sum_{i=0}^{\infty} \frac{(-1)^{i}(\lambda q)^{i}}{i !}\left\{\left(1+\frac{x}{\beta}\right)^{\alpha}-1\right\}^{-i / \sigma} .
$$

Using the same power series as in (4.1), $\rho_{r, q}$ can be expressed as

$$
\rho_{r, q}=\sum_{i, j=0}^{\infty} b_{i, j} \frac{\alpha[(i+1)+\sigma j]}{\beta \sigma} \int_{0}^{\infty} x^{r}\left(1+\frac{x}{\beta}\right)^{-\frac{\alpha}{\sigma}[(i+1)+\sigma j]-1} d x
$$

where

$$
b_{i, j}=\frac{(-1)^{i+j}(q+1)^{i} \lambda^{(i+1)}}{i !((i+1)-\sigma j)}\left(\begin{array}{c}
-\frac{(i+1)}{\sigma}-1 \\
j
\end{array}\right)
$$

Integrating (5.7), we obtain

$$
\rho_{r, q}=\beta^{r} \sum_{i, j=0}^{\infty}-\frac{\alpha}{\sigma}[(i+1)+\sigma j] b_{i, j} B\left((r+1), \frac{\alpha}{\sigma}[(i+1)+\sigma j]-r\right) .
$$




\subsection{Moment generating function}

The mgf $M_{X}(t)$ of $X$ is given by

$$
M_{X}(t)=\sum_{i, j=0}^{\infty} W_{i, j} \frac{\alpha[(i+1)+\sigma j]}{\beta \sigma} \int_{0}^{\infty} \mathrm{e}^{\mathrm{tx}} \underbrace{\left(1+\frac{x}{\beta}\right)^{-\alpha[(i+1)+\sigma j]-1}}_{\mathrm{D}} \mathrm{dx} .
$$

By expanding $D$ in last equation in power series, we obtain

$$
D=\sum_{k=0}^{\infty}\left(\begin{array}{c}
-\alpha[(i+1)+\sigma j]-1 \\
k
\end{array}\right)\left(\frac{x}{\beta}\right)^{k} .
$$

Using the last result in (5.8), the mgf of $X$ follows as

$$
M_{x}(t)=\sum_{i, j, k=0}^{\infty} W_{i, j, k} \frac{\alpha[(i+1)+\sigma j] \Gamma(k+1)}{\beta^{k+1} \sigma t^{k+1}},
$$

where $W_{i, j, k}=\frac{(-1)^{i+j-k-1} \lambda^{(i+1)}}{i !((i+1)-\sigma j)}\left({ }^{-\frac{(i+1)}{\sigma}-1}\right)\left(\begin{array}{c}-\alpha((i+1)+\sigma j)-1 \\ j\end{array}\right)$.

\subsection{Shannon entropy}

Theorem 5.3. The Shannon entropy of $X$ is given by

$$
\eta_{X}=\log \left(\frac{\sigma \beta}{\alpha}\right)+\mu+\xi(\sigma+1)+1-\left(\frac{1}{\alpha}-1\right) \sum_{k=1}^{\infty} V_{k} \Gamma(1-k \sigma),
$$

where $\xi \approx 0.57722$ is the well-known Euler's constant and $V_{k}=\frac{(-1)^{k}}{k} \lambda \sigma k$.

Proof. Based on Al-Aqtash et al. (2014), the Shannon entropy of $X$ is given by

$$
\eta_{X}=\eta_{T}+\mu_{T}-\mathbb{E}\left[\log \left\{f\left(G^{-1}\left\{\mathrm{e}^{\mathrm{T}} /\left(1+\mathrm{e}^{\mathrm{T}}\right)\right\}\right)\right\}\right]-2 \mathbb{E}\left\{\log \left(1+\mathrm{e}^{\mathrm{T}}\right)\right\},
$$

where $\mathrm{T} \sim \operatorname{Gumbel}(\mu, \sigma)$ and $\eta_{T}=\log (\sigma)+\xi+1$ is the Shannon entropy of $T$ (see Nadarajah and Zografos, 2003) and $\mu_{T}=\mu+\xi \sigma$ is the mean of the Gumbel distribution (see Johnson et al., 1995).

First, we consider $\mathbb{E}\left\{\log f\left(G^{-1}\left\{\mathrm{e}^{\mathrm{T}} /\left(1+\mathrm{e}^{\mathrm{T}}\right)\right\}\right)\right\}$, where $f(x)$ and $F(x)$ are the pdf and cdf of the Lomax distribution. Then,

$$
\mathbb{E}\left[\log \left\{f\left[G^{-1}\left\{\mathrm{e}^{\mathrm{T}} /\left(1+\mathrm{e}^{\mathrm{T}}\right)\right\}\right]\right\}\right]=\log \left(\frac{\alpha}{\beta}\right)-\left(\frac{\alpha+1}{\alpha}\right) \mathbb{E}\left[\log \left(1+\mathrm{e}^{\mathrm{T}}\right)\right] .
$$

Inserting the last result in (5.10), we obtain

$$
\eta_{X}=\eta_{T}+\mu_{T}-\log \left(\frac{\alpha}{\beta}\right)+\left(\frac{1}{\alpha}-1\right) \mathbb{E}\left[\log \left(1+\mathrm{e}^{\mathrm{T}}\right)\right] .
$$

Using the generalized binomial expansion, we have

$$
\mathbb{E}\left\{\log \left(1+\mathrm{e}^{\mathrm{T}}\right)\right\}=-\sum_{k=1}^{\infty} \frac{(-1)^{k}}{k} \mathbb{E}\left(\mathrm{e}^{\mathrm{Tk}}\right) .
$$


M.H. Tahir et al.

Thus, equation (5.9) follows by substituting (5.13) in (5.12) and noting that

$$
\mathbb{E}\left(\mathrm{e}^{\mathrm{Tk}}\right)=\lambda^{\sigma k} \Gamma(1-k \sigma) .
$$

Hence, the theorem is proved.

\subsection{Order Statistics}

Order statistics make their appearance in many areas of statistical theory and practice. Suppose $X_{1}, \ldots, X_{n}$ is a random sample from the GuLx distribution. Let $X_{i: n}$ denote the $i$ th order statistic. Then, the pdf of $X_{i: n}$ can be expressed as

$$
\begin{aligned}
f_{i: n}(x) & =\frac{n !}{(i-1) !(n-i) !} f(x) F(x)^{i-1}\{1-F(x)\}^{n-i} \\
& =\frac{n !}{(i-1) !(n-i) !} \sum_{j=0}^{n-i}(-1)^{j}\left(\begin{array}{c}
n-i \\
j
\end{array}\right) f(x) F(x)^{j+i-1} .
\end{aligned}
$$

Inserting (2.4) and (2.5) in the last equation, and after some algebra, we obtain

$$
\begin{aligned}
f_{i: n}(x)= & \sum_{j=0}^{n-i} \frac{(-1)^{j} n !}{(i-1) !(n-i) !}\left(\begin{array}{c}
n-i \\
j
\end{array}\right) \frac{\alpha \lambda\left(1+\frac{x}{\beta}\right)^{-\frac{\alpha}{\sigma}-1}}{\beta \sigma\left\{1-\left(1+\frac{x}{\beta}\right)^{-\alpha}\right\}^{\frac{1}{\sigma}+1}} \\
& \times \exp \left[-(i+j)\left(\lambda\left\{\left(1+\frac{x}{\beta}\right)^{\alpha}-1\right\}^{-1 / \sigma}\right)\right]
\end{aligned}
$$

Hence, the density function of the order statistics can be expressed as

$$
f_{i: n}(x)=\sum_{j=0}^{n-i} \eta_{j} f_{\alpha, \beta, \lambda(i+j), \sigma}(x),
$$

where

$$
\eta_{j}=\frac{(-1)^{j} n !}{(i+j)(i-1) !(n-i) !}\left(\begin{array}{c}
n-i \\
j
\end{array}\right)
$$

and $f_{\alpha, \beta, \lambda(i+j), \sigma}(x)$ is the GuLx density with parameters $(\alpha, \beta, \lambda, \sigma)$. Equation (5.14) is the main result of this section. It reveals that the pdf of the GuLx order statistics is a mixture of GuLx densities. Thus, several mathematical quantities of these order statistics like ordinary and incomplete moments, factorial moments, mgf, mean deviations and several others can be derived from those quantities of the GuLx distribution.

\section{Characterizations of the GuLx distribution}

In designing a stochastic model for a particular modeling problem, an investigator will be vitally interested to know if their model fits the requirements of a specific underlying probability distribution. To this end, the investigator will rely on the characterizations of the selected distribution. Generally speaking, the problem of characterizing a distribution is an important problem in various 
fields and has recently attracted the attention of many researchers. Consequently, various characterization results have been reported in the literature in many different directions. In this section, we present characterizations of the GuLx distribution based on the conditional expectations of certain functions of the random variable.

Proposition 6.1. Let $X: \Omega \rightarrow(0, \infty)$ be a continuous random variable with cdf $F(x)$ and $p d f f(x)$. Let $\psi(x)$ and $q(x)$ be two differentiable functions on $(0, \infty)$ such that $\int_{0}^{\infty} \frac{q^{\prime}(t)}{\psi(t)-q(t)} d t=\infty$. Then,

$$
\mathbb{E}[\psi(X) \mid X<x]=q(x) \quad x>0,
$$

implies

$$
F(x)=\exp \left\{-\int_{x}^{\infty} \frac{q^{\prime}(t)}{\psi(t)-q(t)} d t\right\}, \quad x \geq 0 .
$$

Proof. From equation (6.1), we obtain

$$
\int_{0}^{x} \psi(t) f(t) d t=q(x) F(x) .
$$

Differentiating both sides of the last equation with respect to $x$, we have

$$
\frac{f(x)}{F(x)}=\frac{q^{\prime}(x)}{\psi(x)-q(x)}
$$

or

$$
-\log [F(x)]=\int_{x}^{\infty} \frac{q^{\prime}(t)}{\psi(t)-q(t)} d t
$$

from which we obtain (6.2).

Remark 6.1. (a) Taking, e.g., $\psi(x)=2 q(x)$ and $q(x)=\exp \left\{-\mathrm{e}^{\mu / \sigma}\left[\left(1+\frac{x}{\beta}\right)^{\alpha}-1\right]^{-1 / \sigma}\right\}$, Proposition 6.1 gives a characterization of (2.4).

(b) Clearly there are other pairs of functions $(\psi(x), q(x))$, which satisfy the conditions of Proposition 6.1. We use the one in (a) for simplicity.

Proposition 6.2. Let $X: \Omega \rightarrow(0, \infty)$ be a continuous random variable with $c d f F(x)$ and $p d f$ $f(x)$. Let $\psi_{1}(x)$ and $q_{1}(x)$ be two differentiable functions on $(0, \infty)$ such that $\left[\frac{\psi_{1}^{\prime}(t)-q_{1}^{\prime}(t)}{q_{1}(t)}\right]>0$ and $\int_{0}^{\infty} \frac{\psi_{1}^{\prime}(t)-q_{1}^{\prime}(t)}{q_{1}(t)} d t=\infty$. Then,

$$
\mathbb{E}\left(\psi_{1}(X) \mid X<x\right)=\psi_{1}(x)-q_{1}(x), \quad x>0,
$$

which implies

$$
F(x)=\exp \left\{-\int_{x}^{\infty} \frac{\psi_{1}^{\prime}(t)-q_{1}^{\prime}(t)}{q_{1}(t)} d t\right\}, \quad x>0
$$

Proof. The proof is similar to that of Proposition 6.1. 
Remark 6.2. (c) Taking, e.g., $\psi_{1}(t)=2 q_{1}(x)$ and $q_{1}(x)=\exp \left\{-e^{\mu / \sigma}\left[\left(1+\frac{x}{\beta}\right)^{\alpha}-1\right]^{-1 / \sigma}\right\}$. Proposition 6.2 gives a characterization of (2.4).

(d) Clearly there are other pairs of functions $\left(\psi_{1}(t), q_{1}(x)\right)$ which satisfy conditions of Proposition 6.2. We use the one in (c) for simplicity.

Proposition 6.3. Let $X: \Omega \rightarrow(0, \infty)$ be a continuous random variable with $c d f F(x)$ and $p d f f(x)$. Let $\psi_{2}(x)$ be a differentiable function on $(0, \infty)$ with $\lim _{x \rightarrow 0^{+}} \psi_{2}(x)=\delta>1$ and $\lim _{x \rightarrow \infty} \psi_{2}(x)=\infty$. Then

$$
E\left\{\left[\psi_{2}(X)\right]^{\delta} \mid X<x\right\}=\delta\left[\psi_{2}(x)\right]^{\delta-1}, \quad x>0,
$$

if and only if

$$
\psi_{2}(x)=\delta\left[1+(F(x))^{\frac{1}{\delta-1}}\right]^{-1}, \quad x>0
$$

Proof. The proof is similar to that of Proposition 6.1.

Remark 6.3. (e) Taking, e.g., $\psi_{2}(t)=\delta\left\{1+\left[\exp \left(-\mathrm{e}^{\mu / \sigma}\left[\left(1+\frac{x}{\beta}\right)^{\alpha}-1\right]^{-1 / \sigma}\right)\right]^{\frac{1}{\delta-1}}\right\}^{-1}$. Proposition 6.3 gives a characterization of (2.4).

(f) Clearly there are other functions $\psi_{2}(t)$ which satisfy conditions of Proposition 6.3. We use the one in (e) for simplicity.

\section{Estimation and Information Matrix}

Several approaches for parameter estimation were proposed in the literature but the maximum likelihood method is the most commonly employed. The maximum likelihood estimators (MLEs) enjoy desirable properties and can be used when constructing confidence intervals and regions and also in test statistics. The normal approximation for these estimators in large sample distribution theory is easily handled either analytically or numerically. So, we consider the estimation of the unknown parameters for the GuLx model by maximum likelihood. Let $x_{1}, \ldots, x_{n}$ be a sample of size $n$ from the GuLx distribution given by (2.5).

The log-likelihood function for the vector of parameters $\Theta=(\alpha, \beta, \lambda, \sigma)^{\top}$ can be expressed as

$$
\begin{aligned}
\ell= & n \log \left(\frac{\alpha \lambda}{\beta \sigma}\right)-\left(\frac{\alpha}{\sigma}+1\right) \sum_{i=1}^{n} \log \left(1+\frac{x}{\beta}\right)-\left(\frac{1}{\sigma}+1\right) \sum_{i=1}^{n} \log \left\{1-\left(1+\frac{x}{\beta}\right)^{-\alpha}\right\} \\
& -\lambda \sum_{i=1}^{n}\left\{\left(1+\frac{x}{\beta}\right)^{\alpha}-1\right\}^{-(1 / \sigma)} .
\end{aligned}
$$

Equation (7.1) can be maximized either directly by using the R (optim function), SAS (NLMixed procedure) or Ox (MaxBFGS function), or then by solving the nonlinear likelihood equations by 
differentiating it. The components of the score vector $U(\Theta)$ are given by

$$
\begin{aligned}
U_{\alpha}= & \frac{n}{\alpha}+\frac{1}{\sigma}\left\{n \lambda\left\{\left(1+\frac{x}{\beta}\right)^{\alpha}-1\right\}^{-1-\frac{1}{\sigma}}\left(1+\frac{x}{\beta}\right)^{\alpha} \log \left(1+\frac{x}{\beta}\right)\right\}-\frac{n\left(1+\frac{x}{\beta}\right)^{\alpha}\left(1+\frac{1}{\sigma}\right)}{\left(1+\frac{x}{\beta}\right)^{\alpha}-1} \\
& \left\{\log \left(1+\frac{x}{\beta}\right)-\left\{\left(1+\frac{x}{\beta}\right)^{\alpha}-1\right\}\left(1+\frac{x}{\beta}\right)^{-\alpha} \log \left(1+\frac{x}{\beta}\right)\right\}-\frac{n}{\sigma} \log \left(1+\frac{x}{\beta}\right), \\
U_{\beta}= & -\frac{n}{\beta}+\frac{n}{\left\{\left(1+\frac{x}{\beta}\right)^{\alpha}-1\right\} \beta^{2}}\left\{\frac{x \alpha}{\left(1+\frac{x}{\beta}\right)}-x \alpha\left\{\left(1+\frac{x}{\beta}\right)^{\alpha}-1\right\}\left(1+\frac{x}{\beta}\right)^{-\alpha-1}\right\}\left(1+\frac{x}{\beta}\right)^{\alpha}\left(1+\frac{1}{\sigma}\right) \\
& -\frac{n}{x+\beta}\left\{1-\left(1+\frac{x}{\beta}\right)\right\}\left(1+\frac{\alpha}{\sigma}\right)-\frac{n x \alpha \lambda}{\beta^{2} \sigma}\left\{\left(1+\frac{x}{\beta}\right)^{\alpha}-1\right\}^{-\frac{1}{\sigma}-1}\left(1+\frac{x}{\beta}\right)^{\alpha-1}, \\
U_{\lambda}= & \frac{n}{\lambda}-n\left\{\left(1+\frac{x}{\beta}\right)^{\alpha}-1\right\}^{-1 / \sigma}, \\
U_{\sigma}= & -\frac{n}{\sigma}-\frac{n \lambda}{\sigma^{2}}\left\{\left(1+\frac{x}{\beta}\right)^{\alpha}-1\right\}^{-1 / \sigma} \log \left[\left(1+\frac{x}{\beta}\right)^{\alpha}-1\right]+\frac{n \alpha}{\sigma^{2}} \log \left(1+\frac{x}{\beta}\right) \\
& +\frac{n}{\sigma^{2}} \log \left[\left(1+\frac{x}{\beta}\right)^{-\alpha}\left\{\left(1+\frac{x}{\beta}\right)^{\alpha}-1\right\} .\right.
\end{aligned}
$$

Setting these equations to zero and solving them simultaneously yields the MLEs of the model parameters.

For interval estimation of the parameters, we require the $4 \times 4$ observed information matrix $J(\Theta)=\left\{-J_{r s}\right\}$ (for $r, s=\alpha, \beta, \lambda, \sigma$ ). Standard maximization routines provide only an approximation for the observed information matrix, which is given in Appendix A.

Under standard regularity conditions, the multivariate normal $N_{4}\left(0, J(\widehat{\Theta})^{-1}\right)$ distribution can be used to construct approximate confidence intervals for the model parameters. Here, $J(\widehat{\Theta})$ is the total observed information matrix evaluated at $\widehat{\Theta}$. Then, the $100(1-\gamma) \%$ confidence intervals for $\alpha, \beta, \mu$ and $\sigma$ are given by $\hat{\alpha} \pm z_{\gamma^{*} / 2} \times \sqrt{\operatorname{var}(\hat{\alpha})}, \hat{\beta} \pm z_{\gamma^{*} / 2} \times \sqrt{\operatorname{var}(\hat{\beta})}, \hat{\lambda} \pm z_{\gamma^{*} / 2} \times \sqrt{\operatorname{var}(\hat{\lambda})}$ and $\hat{\sigma} \pm z_{\gamma^{*} / 2} \times \sqrt{\operatorname{var}(\hat{\sigma})}$, respectively, where the $\operatorname{var}(\cdot)$ 's denote the diagonal elements of $J(\widehat{\Theta})^{-1}$ corresponding to the model parameters, and $z_{\gamma^{*} / 2}$ is the quantile $\left(1-\gamma^{*} / 2\right)$ of the standard normal distribution.

\subsection{A simulation study}

We evaluate the performance of the maximum likelihood method for estimating the GuLx parameters using Monte Carlo simulation for a total of eight parameter combinations. Two different sample sizes $n=100$ and 300 are considered. The process is repeated 300 times in order to obtain average estimates (AEs), biases and mean square errors (MSEs) of the parameters. They are listed in Table 1. The small values of the biases and MSEs indicate that the maximum likelihood method performs quite well in estimating the model parameters of the proposed distribution. 
Table 1: Estimated AEs, Biases, and MSEs of the MLEs of parameters of GuLx distribution based on 300 simulations of with $n=100$ and 300 .

\begin{tabular}{|c|c|c|c|c|c|c|c|c|c|c|c|c|c|c|c|c|}
\hline \multirow[b]{2}{*}{$n$} & \multicolumn{4}{|c|}{ Actual values } & \multicolumn{4}{|c|}{$\mathrm{AE}$} & \multicolumn{4}{|c|}{ Bias } & \multicolumn{4}{|c|}{ MSE } \\
\hline & $\alpha$ & $\beta$ & $\sigma$ & $\lambda$ & $\tilde{\alpha}$ & $\tilde{\beta}$ & $\tilde{\sigma}$ & $\tilde{\lambda}$ & $\tilde{\alpha}$ & $\tilde{\beta}$ & $\tilde{\sigma}$ & $\tilde{\lambda}$ & $\tilde{\alpha}$ & $\tilde{\beta}$ & $\tilde{\sigma}$ & $\tilde{\lambda}$ \\
\hline \multirow[t]{4}{*}{100} & 0.5 & 1.0 & 0.5 & 1.0 & 0.553 & 0.514 & 1.100 & 1.071 & 0.053 & 0.014 & 0.100 & 0.071 & 0.053 & 0.008 & 0.188 & 0.121 \\
\hline & 0.5 & 1.0 & 1.5 & 1.0 & 0.515 & 1.473 & 0.950 & 1.097 & 0.015 & -0.027 & -0.050 & 0.097 & 0.033 & 0.071 & 0.180 & 0.173 \\
\hline & 1.5 & 2.0 & 0.5 & 1.5 & 1.631 & 0.504 & 1.847 & 1.413 & 0.131 & 0.004 & -0.153 & -0.087 & 0.343 & 0.005 & 0.327 & 0.175 \\
\hline & 1.5 & 2.0 & 2.5 & 1.5 & 1.648 & 2.581 & 2.272 & 1.130 & 0.148 & 0.081 & 0.272 & -0.370 & 0.236 & 0.181 & 1.909 & 0.377 \\
\hline \multirow[t]{4}{*}{300} & 0.5 & 1.0 & 0.5 & 1.0 & 0.522 & 0.507 & 1.045 & 1.036 & 0.022 & 0.007 & 0.045 & 0.036 & 0.009 & 0.002 & 0.047 & 0.031 \\
\hline & 0.5 & 1.0 & 1.5 & 1.0 & 0.498 & 1.479 & 0.967 & 1.038 & -0.002 & -0.021 & -0.033 & 0.038 & 0.010 & 0.023 & 0.094 & 0.054 \\
\hline & 1.5 & 2.0 & 0.5 & 1.5 & 1.618 & 0.512 & 1.856 & 1.417 & 0.118 & 0.012 & -0.144 & -0.083 & 0.145 & 0.002 & 0.148 & 0.077 \\
\hline & 1.5 & 2.0 & 2.5 & 1.5 & 1.522 & 2.518 & 2.518 & 1.095 & 0.022 & 0.018 & 0.043 & -0.405 & 0.050 & 0.051 & 0.863 & 0.305 \\
\hline
\end{tabular}

\section{Applications}

In this section, we provide two applications to real data sets to illustrate the flexibility of the GuLx distribution. The model parameters are estimated by maximum likelihood and three goodness-of-fit statistics are used to compare this distribution with three other models, namely: the gamma-Lomax (GaLx) (Cordeiro et al., 2015), exponentiated Lomax (ELx) (Abdul-Moniem and Abdel-Hameed, 2012) and beta-Lomax (BLx) (Lemonte and Cordeiro, 2013). Their associated density functions are, respectively, given by:

$$
\begin{aligned}
& f_{G a L x}(x ; a, \alpha, \beta)=\frac{\alpha^{a}}{\beta \Gamma(a)}\left(1+\frac{x}{\beta}\right)^{-(\alpha+1)}\left\{\log \left[1+\frac{x}{\beta}\right]\right\}^{a-1}, \\
& f_{E L x}(x ; a, \alpha, \beta)=\frac{a \alpha}{\beta}\left(1+\frac{x}{\beta}\right)^{-(k+1)}\left\{1-\left(1+\frac{x}{\beta}\right)^{-k}\right\}^{a-1},
\end{aligned}
$$

and

$$
f_{B L x}(x ; a, b, \alpha, \beta)=\frac{\alpha}{\beta B(a, b)}\left(1+\frac{x}{\beta}\right)^{-(\alpha b+1)}\left\{1-\left(1+\frac{x}{\beta}\right)^{-\alpha}\right\}^{a-1} .
$$

We generally apply one or more goodness-of-fit tests to see how well a selected distribution fits to the lifetime data. The goodness-of-fit statistics including Akaike information criterion (AIC), Anderson-Darling $\left(A^{*}\right)$, Cramér-von Mises $\left(W^{*}\right)$ and Kolmogrov-Smirnov (K-S) are computed to compare the fitted models. The statistics $A^{*}$ and $W^{*}$ are described by Chen and Balakrishnan (1995). The K-S statistic compares the empirical cumulative distribution of the data to any specified continuous distribution when its parameters are estimated by maximum likelihood. This comparison of the two cdfs looks only at the point of maximum discrepancy of this statistic. The lower are the K-S values more evidence we have that the specified model generates the data. More details of this test are described by Evans et al. (2008). All computations are carried out using the R-software.

The first real data set is a subset of the data reported by Bekker et al. (2000), which corresponds to the survival times (in years) of a group of patients given chemotherapy treatment alone. The data consisting of survival times (in years) for 46 patients are: $0.047,0.115,0.121,0.132,0.164,0.197$, $0.203,0.260,0.282,0.296,0.334,0.395,0.458,0.466,0.501,0.507,0.529,0.534,0.540,0.641$, $0.644,0.696,0.841,0.863,1.099,1.219,1.271,1.326,1.447,1.485,1.553,1.581,1.589,2.178$, $2.343,2.416,2.444,2.825,2.830,3.578,3.658,3.743,3.978,4.003,4.033$. The second real data consists of the number of successive failure for the air conditioning system reported of each member 
in a fleet of 13 Boeing 720 jet airplanes. The pooled data with 214 observations was considered by Proschan (1963), Kus (2007) and many others. The data are: 50, 130, 487, 57, 102, 15, 14, 10, 57, $320,261,51,44,9,254,493,33,18,209,41,58,60,48,56,87,11,102,12,5,14,14,29,37,186$, $29,104,7,4,72,270,283,7,61,100,61,502,220,120,141,22,603,35,98,54,100,11,181,65$, $49,12,239,14,18,39,3,12,5,32,9,438,43,134,184,20,386,182,71,80,188,230,152,5,36$, $79,59,33,246,1,79,3,27,201,84,27,156,21,16,88,130,14,118,44,15,42,106,46,230,26$, $59,153,104,20,206,5,66,34,29,26,35,5,82,31,118,326,12,54,36,34,18,25,120,31,22$, $18,216,139,67,310,3,46,210,57,76,14,111,97,62,39,30,7,44,11,63,23,22,23,14,18$, $13,34,16,18,130,90,163,208,1,24,70,16,101,52,208,95,62,11,191,14,71$.

Table 2: MLEs and their standard errors (in parentheses) for the fitted models.

\begin{tabular}{|c|c|c|c|c|c|c|}
\hline Distribution & $a$ & $b$ & $\alpha$ & $\beta$ & $\lambda$ & $\sigma$ \\
\hline \multicolumn{7}{|c|}{ Data 1} \\
\hline GuLx (3p) & $\begin{array}{l}- \\
-\end{array}$ & - & $\begin{array}{c}59.4419 \\
(06.0974)\end{array}$ & $\begin{array}{c}46.7872 \\
(04.9685)\end{array}$ & $\begin{array}{c}1.0000 \\
-\end{array}$ & $\begin{array}{c}1.7289 \\
(0.2014)\end{array}$ \\
\hline GuLx (4p) & $\begin{array}{l}- \\
-\end{array}$ & - & $\begin{array}{c}62.2930 \\
(19.4718)\end{array}$ & $\begin{array}{c}42.5706 \\
(06.0975)\end{array}$ & $\begin{array}{c}1.1634 \\
(0.4644)\end{array}$ & $\begin{array}{c}1.8454 \\
(0.4035)\end{array}$ \\
\hline GaLx & $\begin{array}{c}1.1525 \\
(0.2423)\end{array}$ & - & $\begin{array}{c}17.1850 \\
(03.0668)\end{array}$ & $\begin{array}{c}18.7869 \\
(04.0444)\end{array}$ & $\begin{array}{l}- \\
-\end{array}$ & $\begin{array}{l}- \\
-\end{array}$ \\
\hline Elx & $\begin{array}{l}1.1340 \\
(0.2428)\end{array}$ & $\begin{array}{l}- \\
-\end{array}$ & $\begin{array}{c}30.2458 \\
(04.2220)\end{array}$ & $\begin{array}{c}36.2757 \\
(05.9303)\end{array}$ & - & $\begin{array}{l}- \\
-\end{array}$ \\
\hline BLx & $\begin{array}{c}1.1194 \\
(0.2210)\end{array}$ & $\begin{array}{c}11.5367 \\
(08.4681)\end{array}$ & $\begin{array}{c}4.1860 \\
(05.7002)\end{array}$ & $\begin{array}{c}56.9449 \\
(03.3815)\end{array}$ & - & - \\
\hline \multicolumn{7}{|c|}{ Data 2} \\
\hline GuLx (3p) & $\begin{array}{l}- \\
-\end{array}$ & - & $\begin{array}{l}21.9694 \\
(1.7949)\end{array}$ & $\begin{array}{c}995.0593 \\
(7.9947)\end{array}$ & $\begin{array}{c}1.0000 \\
-\end{array}$ & $\begin{array}{c}1.9508 \\
(0.1236)\end{array}$ \\
\hline GuLx (4p) & $\begin{array}{l}- \\
-\end{array}$ & - & $\begin{array}{c}5.8043 \\
(2.2384)\end{array}$ & $\begin{array}{l}32.4258 \\
(2.9737)\end{array}$ & $\begin{array}{c}3.4083 \\
(0.2117)\end{array}$ & $\begin{array}{c}3.3098 \\
(0.5940)\end{array}$ \\
\hline GaLx & $\begin{array}{c}1.2253 \\
(0.2190)\end{array}$ & - & $\begin{array}{c}3.1831 \\
(1.3108)\end{array}$ & $\begin{array}{l}156.1894 \\
(15.5067)\end{array}$ & $\begin{array}{l}- \\
-\end{array}$ & $\begin{array}{l}- \\
-\end{array}$ \\
\hline Elx & $\begin{array}{l}1.2176 \\
(0.2111)\end{array}$ & $\begin{array}{l}- \\
-\end{array}$ & $\begin{array}{c}3.1413 \\
(1.4100)\end{array}$ & $\begin{array}{l}170.7597 \\
(19.3842)\end{array}$ & $\begin{array}{l}- \\
-\end{array}$ & - \\
\hline BLx & $\begin{array}{c}1.6939 \\
(0.7095)\end{array}$ & $\begin{array}{c}0.0459 \\
(0.0465)\end{array}$ & $\begin{array}{l}102.2161 \\
(33.2301)\end{array}$ & $\begin{array}{l}319.1521 \\
(41.9613)\end{array}$ & $\begin{array}{l}- \\
-\end{array}$ & - \\
\hline
\end{tabular}

Table 3: The statistics $\hat{\ell}, \mathrm{AIC}, A^{*}, W^{*}$ and $\mathrm{K}-\mathrm{S}$ for the data sets $1 \& 2$.

\begin{tabular}{lcccccc}
\hline Distribution & $\hat{\ell}$ & AIC & $A^{*}$ & $W^{*}$ & K-S & p-value \\
\hline \multicolumn{7}{c}{ Data set 1} \\
\hline GuLx (3p) & 56.8715 & 119.7429 & 0.3040 & 0.0392 & 0.0710 & 0.9651 \\
GuLx (4p) & 56.8025 & 121.6050 & 0.3152 & 0.0420 & 0.0748 & 0.9463 \\
GaLx & 58.1850 & 122.3701 & 0.5118 & 0.0761 & 0.1087 & 0.6229 \\
Elx & 58.1557 & 122.3113 & 0.5152 & 0.0766 & 0.1079 & 0.6324 \\
BLx & 58.1277 & 124.2554 & 0.5221 & 0.0777 & 0.1095 & 0.6144 \\
\hline & \multicolumn{7}{c}{ Data set 2 } \\
\hline GuLx (3p) & 977.7956 & 1961.5910 & 0.3240 & 0.0419 & 0.0481 & 0.8030 \\
GuLx (4p) & 981.0735 & 1970.1470 & 0.5924 & 0.0835 & 0.0490 & 0.7833 \\
GaLx & 979.0003 & 1964.0010 & 0.4510 & 0.0654 & 0.0488 & 0.7874 \\
Elx & 979.0217 & 1964.0430 & 0.4568 & 0.0664 & 0.0489 & 0.7860 \\
BLx & 979.0424 & 1966.0850 & 0.4252 & 0.0602 & 0.0487 & 0.7894 \\
\hline
\end{tabular}

Table 2 lists the MLEs and their corresponding standard errors (in parentheses) of the estimates of the parameters for the fitted models. The statistics $(\hat{\ell}), \mathrm{AIC}, A^{*}, W^{*}$ and $\mathrm{K}-\mathrm{S}$ are listed in Table 3. For the first data set, the GuLx model with 3-parameters and 4-parameters provide the best fits. 
(a) Estimated pdfs for data set 1

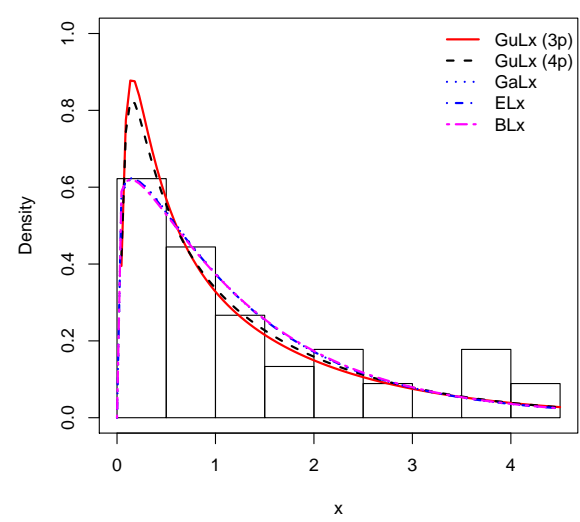

(c) Estimated pdfs for data set 2

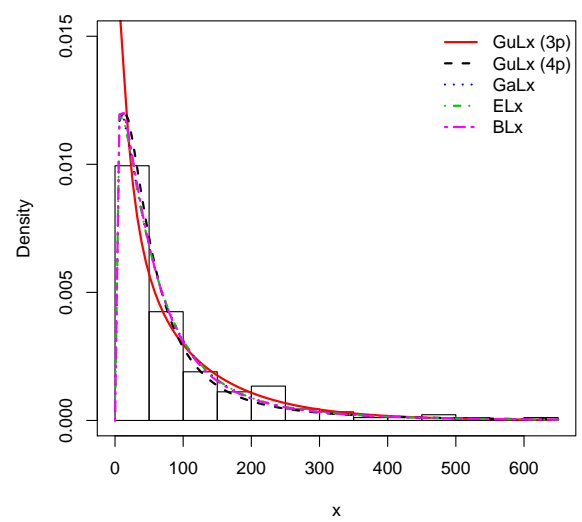

(b) Estimated cdfs for data set 1

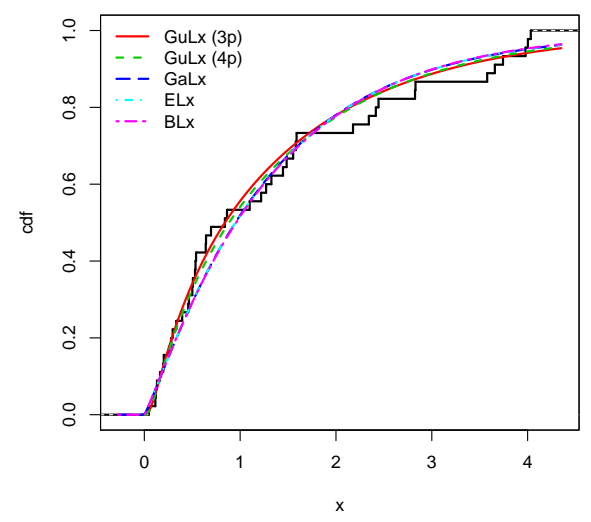

(d) Estimated cdfs for data set 2

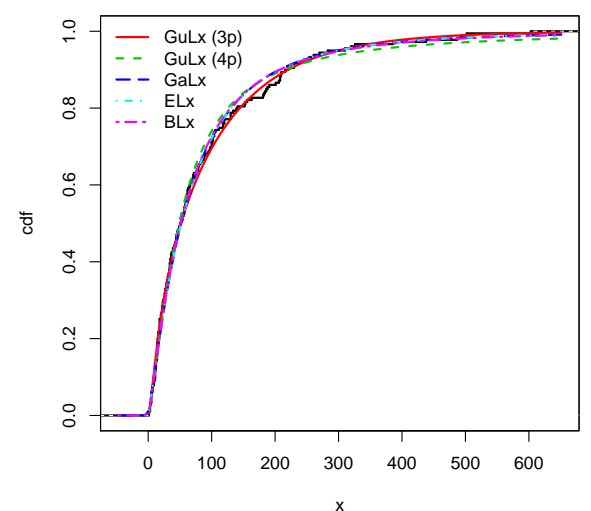

Fig. 3. Plots of the estimated pdfs and cdfs of the GuLx, GaLx, ELx, BLx and McLx models for data sets 1 \& 2.

For the second data set, the GuLx model with 3-parameters provides the best fit. The empirical pdfs and cdfs are displayed in Figure 3, which support the results of Table 3.

\section{Concluding remarks}

The interest in developing more flexible statistical distributions remains strong in statistical theory and applications. Many generalized classes of distributions have been developed and applied to describe various phenomena. In this paper, we propose a new lifetime model called the GumbelLomax (GuLx) distribution. We study some of its structural properties including an expansion for the density function and explicit expressions for the ordinary and incomplete moments, generating function, mean deviations, quantile function, order statistics and Shannon entropy. The maximum likelihood method is employed for estimating the model parameters and the observed information matrix is determined. We fit the new model to two real data sets to demonstrate its usefulness in 
practice. We conclude that the GuLx model provides consistently better fits than other competing models for these data sets. We hope that the proposed model will attract wider applications in areas such as engineering, survival and lifetime data, hydrology, economics, among others.

\section{Appendix A}

The observed information matrix for the parameter vector $\Theta=(\alpha, \beta, \lambda, \sigma)^{\top}$ is given by

$$
J(\Theta)=-\frac{\partial^{2} \ell(\Theta)}{\partial \Theta \partial \Theta^{\top}}=-\left(\begin{array}{cccc}
J_{\alpha \alpha} & J_{\alpha \beta} & J_{\alpha \lambda} & J_{\alpha \sigma} \\
\cdot & J_{\beta \beta} & J_{\beta \lambda} & J_{\beta \sigma} \\
\cdot & \cdot & J_{\lambda, \lambda} & J_{\lambda \sigma} \\
\cdot & \cdot & \cdot & J_{\sigma \sigma}
\end{array}\right)
$$

whose elements are

$$
\begin{aligned}
& J_{\alpha \alpha}=-\frac{1}{\alpha^{2} \sigma^{2}} n\left\{\left(1+\frac{x}{\beta}\right)^{\alpha}-1\right\}^{-\frac{1}{\sigma}-2}\left[\sigma^{2}\left\{\left(1+\frac{x}{\beta}\right)^{\alpha}-1\right\}^{\frac{1}{\sigma}+2}+\alpha^{2}\left(1+\frac{x}{\beta}\right)^{\alpha}\right. \\
& \left.\times\left\{\left[-\sigma(1+\sigma)\left\{\left(1+\frac{x}{\beta}\right)^{\alpha}-1\right\}^{\frac{1}{\sigma}}+\lambda\left(\left(1+\frac{x}{\beta}\right)^{\alpha}+\sigma\right)\right] \log \left[1+\frac{x}{\beta}\right]^{2}\right\}\right], \\
& J_{\alpha \beta}=\frac{n x}{\beta(x+\beta) \sigma^{2}}\left\{\left(1+\frac{x}{\beta}\right)^{\alpha}-1\right\}^{-\frac{1}{\sigma}-2}\left[-\left\{\left(1+\frac{x}{\beta}\right)^{\alpha}-1\right\} \sigma\left\{\lambda\left(1+\frac{x}{\beta}\right)^{\alpha}\right.\right. \\
& \left.-\left\{\left(1+\frac{x}{\beta}\right)^{\alpha}-1\right\}^{\frac{1}{\sigma}}\left[\left(1+\frac{x}{\beta}\right)^{\alpha}+\sigma\right]\right\}+\alpha\left(1+\frac{x}{\beta}\right)^{\alpha}\left[-\left\{\left(1+\frac{x}{\beta}\right)^{\alpha}-1\right\}^{\frac{1}{\sigma}}\right. \\
& \left.\left.\times \sigma(1+\sigma)+\lambda\left\{\left(1+\frac{x}{\beta}\right)^{\alpha}+\sigma\right\}\right] \log \left[1+\frac{x}{\beta}\right]\right], \\
& J_{\alpha \lambda}=\frac{n}{\sigma}\left\{\left(1+\frac{x}{\beta}\right)^{\alpha}-1\right\}^{-\frac{1}{\sigma}-1}\left(1+\frac{x}{\beta}\right)^{\alpha} \log \left[1+\frac{x}{\beta}\right] \text {, } \\
& \left.J_{\alpha \sigma}=\frac{n}{\sigma^{3}}\left(1+\frac{x}{\beta}\right)^{\alpha}\left\{\left(1+\frac{x}{\beta}\right)^{\alpha}-1\right\}^{-\frac{1+\sigma}{\sigma}} \log \left[1+\frac{x}{\beta}\right]\right\}\left\{\sigma \left[\left\{\left(1+\frac{x}{\beta}\right)^{\alpha}-1\right\}^{\frac{1}{\sigma}}\right.\right. \\
& \left.-\lambda]+\lambda \log \left[\left(1+\frac{x}{\beta}\right)^{\alpha}-1\right]\right\} \\
& J_{\beta \beta}=\frac{n\left\{\left(\frac{\beta+x}{\beta}\right)^{\alpha}-1\right\}^{-\frac{1}{\sigma}-2}}{\beta^{2} \sigma^{2}(\beta+x)^{2}}\left[\sigma ( \frac { \beta + x } { \beta } ) ^ { \alpha } \left[\{ ( \frac { \beta + x } { \beta } ) ^ { \alpha } - 1 \} ^ { 1 / \sigma } \left\{-2 \beta^{2} \sigma+\alpha x^{2}\{(\alpha-1) \sigma\right.\right.\right. \\
& +\alpha+1\}-2 \alpha \beta(\sigma-1) x\}-\alpha \lambda x(2 \beta+\alpha x+x)]+\sigma^{2}\left(\beta^{2}+\alpha x^{2}+2 \alpha \beta x\right)\left\{\left(\frac{\beta+x}{\beta}\right)^{\alpha}-1\right\}^{1 / \sigma} \\
& \left.+\left(\frac{\beta+x}{\beta}\right)^{2 \alpha}\left\{\sigma\left(\beta^{2} \sigma-\alpha x(2 \beta+x)\right)\left\{\left(\frac{\beta+x}{\beta}\right)^{\alpha}-1\right\}^{1 / \sigma}+\alpha \lambda x(2 \beta \sigma+x(\sigma-\alpha))\right\}\right], \\
& J_{\beta \lambda}=-\frac{n x \alpha}{\beta^{2} \sigma}\left(1+\frac{x}{\beta}\right)^{\alpha-1}\left\{\left(1+\frac{x}{\beta}\right)^{\alpha}-1\right\}^{-\frac{1+\sigma}{\sigma}} \text {, }
\end{aligned}
$$




$$
\begin{aligned}
J_{\beta \sigma}= & -\frac{n x \alpha}{\beta^{2} \sigma^{3}}\left(1+\frac{x}{\beta}\right)^{-1+\alpha}\left\{\left(1+\frac{x}{\beta}\right)^{\alpha}-1\right\}^{-\frac{1+\sigma}{\sigma}}\left\{\sigma\left[\left\{\left(1+\frac{x}{\beta}\right)^{\alpha}-1\right\}^{\frac{1}{\sigma}}-\lambda\right]\right. \\
& +\lambda \log \left[\left(1+\frac{x}{\beta}\right)^{\alpha}-1\right], \\
J_{\lambda \lambda}= & -\frac{n}{\lambda^{2}}, \\
J_{\lambda \sigma}= & -\frac{n}{\sigma^{2}}\left\{\left(1+\frac{x}{\beta}\right)^{\alpha}-1\right\}^{-1 / \sigma} \log \left[\left(1+\frac{x}{\beta}\right)^{\alpha}-1\right], \\
J_{\sigma \sigma}= & \frac{n}{\sigma^{4}}\left\{\sigma^{2}+\lambda\left\{\left(\frac{\beta+x}{\beta}\right)^{\alpha}-1\right\}^{-1 / \sigma} \log \left\{\left(\frac{\beta+x}{\beta}\right)^{\alpha}-1\right\}\left\{2 \sigma-\log \left[\left(\frac{\beta+x}{\beta}\right)^{\alpha}-1\right]\right\}\right. \\
& \left.-2 \sigma\left\{\log \left[1-\left(\frac{\beta+x}{\beta}\right)^{-\alpha}\right]+\alpha \log \left[\frac{\beta+x}{\beta}\right]\right\}\right\}^{\alpha} .
\end{aligned}
$$

\section{Acknowledgments}

The authors are thankful to the Editor and the two anonymous referees for their valuable suggestions which helped in improving an earlier version of this paper.

\section{References}

[1] Abdul-Moniem, I.B. and Abdel-Hameed, H.F. (2012). On exponentiated Lomax distribution. International Journal of Mathematical Archive, 3, 2144-2150.

[2] Al-Aqtash, R. (2013). On Generating a New Family of Distributions Using the Logit Function. Ph.D thesis, Central Michigan University Mount Pleasant, Michigan. http://condor.cmich.edu/cdm/ref/collection/p1610-01coll1/id/3817

[3] Al-Aqtash, R., Lee, C. and Famoye, F. (2014). Gumbel-Weibull distribution: Properties and applications. Journal of Modern Applied Statistical Methods, 13, 201-225.

[4] Al-Aqtash, R., Famoye, F. and Lee, C. (2015). On generating a new family of distributions using the logit function. Journal of Probability and Statistical Science, Still in press.

[5] Alexander, C., Cordeiro, G.M., Ortega, E.M.M. and Sarabia, J.M. (2012). Generalized beta-generated distributions. Computational Statistics and Data Analysis, 56, 1880-1897.

[6] Alzaatreh, A. (2011). New Method for Generating Families of Continuous Distributions. Ph.D. thesis, Central Michigan University, Mount Pleasant, Michigan.

http://condor.cmich.edu/cdm/ref/collection/p1610-01coll1/id/3447

[7] Alzaatreh, A., Lee, C. and Famoye, F. (2013). A new method for generating families of continuous distributions. Metron, 71, 63-79.

[8] Amini, M., MirMostafaee, S.M.T.K. and Ahmadi, J. (2014). Log-gamma-generated families of distributions. Statistics, 48, 913-932.

[9] Azzalini, A. (1985). A class of distributions which includes the normal ones. Scandinavian Journal of Statistics, 12, 171-178.

[10] Bekker, A., Roux, J. and Mostert, P. (2000). A generalization of the compound Rayleigh distribution: using a Bayesian methods on cancer survival times. Communications in Statistics-Theory and Methods, 29, 1419-1433.

[11] Bourguignon, M., Silva, R.B. and Cordeiro, G.M. (2014). The Weibull-G family of probability distributions. Journal of Data Science, 12, 53-68.

[12] Chen, G and Balakrishnan, N. (1995). A general purpose approximate goodness-of-fit test. Journal of Quality Technology, 27, 154-161.

[13] Cordeiro, G.M. and de Castro, M. (2011). A new family of generalized distributions. Journal of Statistical Computation and Simulation, 81, 883-893. 
[14] Cordeiro, G.M., Ortega, E.M.M. and da Cunha, D.C.C. (2013). The exponentiated generalized class of distributions. Journal of Data Science, 11, 1-27.

[15] Cordeiro, G.M., Ortega, E.M.M., Popović, B.V. and Pescim, R.R. (2014). The Lomax generator of distributions: Properties, minification process and regression model. Applied Mathematics and Computation, 247, 465-486.

[16] Cordeiro, G.M., Ortega, E.M.M. and Popović, B.V. (2015). The gamma-Lomax distribution. Journal of Statistical Computation and Simulation, 85, 305-319.

[17] Eugene, N., Lee, C. and Famoye, F. (2002). Beta-normal distribution and its applications. Communications in Statistics-Theory and Methods, 31, 497-512.

[18] Evans, D. L., Drew, J. H. and Leemis, L. M. (2008). The distribution of the Kolmogorov-Smirnov, Cramer-von Mises, and Anderson-Darling test statistics for exponential populations with estimated parameters. Communications in Statistics, Simulation and Computation, 37, 1396-1421.

[19] Gross, A. J. and Clark, V. A. (1975). Survival Distributions: Reliability Applications in the Biomedical Sciences. Wiley, New York.

[20] Gupta, R.C., Gupta, P.I. and Gupta, R.D. (1998). Modeling failure time data by Lehmann alternatives. Communications in Statistics-Theory and Method, 27, 887-904.

[21] Johnson, N.L., Kotz, S. and Balakrishnan, N. (1995). Continuous Univariate Distributions. Vol. 2 (2nd edition), Wiley, New York.

[22] Jones, M.C. (2004). Families of distributions arising from the distributions of order statistics. Test, 13, $1-43$.

[23] Kus, C. (2007). A new lifetime distribution. Computational Statistics and Data Analysis, 51, 44974509.

[24] Lemonte, A. J. and Cordeiro, G. M. (2013). An extended Lomax distribution. Statistics, 47, 800-816.

[25] Marshall, A.N. and Olkin, I. (1997). A new method for adding a parameter to a family of distributions with applications to the exponential and Weibull families. Biometrika, 84, 641-652.

[26] Nadarajah, S. and Zografos, K. (2003). Formulas for Rényi information and related measures for univariate distributions. Information Sciences, 155, 119-138.

[27] Proschan, F. (1963). Theoretical explanation of observed decreasing failure rate. Technometrics, 5, 375383.

[28] Ristić, M.M. and Balakrishnan, N. (2012). The gamma-exponentiated exponential distribution. Journal of Statistical Computation and Simulation, 82, 1191-1206.

[29] Torabi, H. and Montazari, N.H. (2012). The gamma-uniform distribution and its application. Kybernetika, 48, 16-30.

[30] Torabi, H. and Montazari, N.H. (2014). The logistic-uniform distribution and its application. Communications in Statistics-Simulation and Computation, 43, 2551-2569.

[31] Tahir, M.H., Cordeiro, G.M., Alzaatreh, A., Mansoor, M. and Zubair, M. (2015a). The Logistic-X family of distributions and its applications. Communications in Statistics-Theory and Methods, forthcoming.

[32] Tahir, M.H., Cordeiro, G.M., Alizadeh, M., Mansoor, M., Zubair M., Hamedani, G.G., (2015b). The odd generalized exponential family of distributions with applications. Journal of Statistical Distributions and Applications, 2, Article 1, DOI:10.1186/s40488-014-0024-2

[33] Tahir, M.H., Zubair, M., Mansoor, M., Cordeiro, G.M., Alizadeh, M. and Hamedani. G.G. (2015c). A new Weibull-G family of distributions. Hacettepe Journal of Mathematics and Statistics, to appear. Doi: 10.15672/HJMS.2015579686

[34] Zografos K. and Balakrishnan, N. (2009). On families of beta- and generalized gamma generated distributions and associated inference. Statistical Methodology, 6, 344-362. 